A. Azzam and E. Kreyszig

Nagoya Math. J.

Vol. 84 (1981), 159-168

\title{
SMOOTHNESS OF SOLUTIONS OF PARABOLIC EQUATIONS IN REGIONS WITH EDGES
}

\author{
A. AZZAM AND E. KREYSZIG*
}

\section{§1. Introduction}

We consider the mixed initial-boundary value problem for the parabolic equation

$$
L u=\sum_{i, j=1}^{2} a_{i j}(x, t) u_{x_{i} x_{j}}+\sum_{j=1}^{2} a_{j}(x, t) u_{x_{j}}+b(x, t) u-u_{t}=f(x, t)
$$

in a region $\Omega \times(0, T]$, where $x=\left(x_{1}, x_{2}\right)$ and $\Omega \subset R^{2}$ is a simply-connected bounded domain having corners.

Our main objective will be the study of smoothness properties of solutions of that problem. Early investigations of that type concern elliptic equations in domains with a smooth boundary, starting with the Dirichlet problem for the Laplace and Poisson equations and proceeding to general second order elliptic equations as well as general boundary conditions; see S. Agmon, A. Douglis and L. Nirenberg [1]. A more recent survey of the elliptic case and further references are given by D. Gilbarg and N. S. Trudinger [6].

Similar work on parabolic equations appeared later; we mention in particular investigations by A. Friedman [5] on the first boundary value problem, by Z. Itô [8] and L. I. Kamynin and V. N. Maslennikova [9] on the second boundary value problem and by N. V. Zitaraŝu [15] on general boundary value problems. Further references are given in [12].

The case of a nonsmooth boundary was treated by E. A. Volkov [14], V. A. Kondrat'ev [10] and others whose work is discussed or mentioned in [7]; all these papers concern elliptic equations, whereas we shall deal with parabolic equations.

We want to mention that those problems in regions with edges and

Received December 21, 1979.

* Work supported by the N.S.E.R.C. of Canada under grant no. A9097. 
corners are also of practical importance in applications, for instance in heat flow (cf. [4]), mechanics of continua and numerical analysis (difference methods, subtraction of singularities, acceleration of convergence; cf. [11], which treats elliptic equations but involves ideas which are also relevant in connection with parabolic equations).

It is known that in the case of a smooth boundary $\partial \Omega$ of $\Omega$, the smoothness of solutions increases with that of the coefficients of (1.1) and the boundary data. Indeed, if $\partial \Omega$ is of class $C^{2+\alpha}, 0<\alpha<1$, the coefficients of (1.1) are of class $C^{\alpha}(\bar{G}), G=\Omega \times(0, T]$, and $u$ is a solution of (1.1) in $G$ satisfying

$$
\begin{aligned}
& u(x, 0)=0 \quad \text { on } \bar{\Omega} \\
& \beta(x, t) u+\eta(x, t) u_{n}=0 \quad \text { on } \partial \Omega \times(0, T],
\end{aligned}
$$

where $u_{n}$ is the outer normal derivative and $\beta \in C^{2+a}(\partial \Omega \times(0, T]), \eta \in$ $C^{1+\alpha}(\partial \Omega \times(0, T])$, then $u \in C^{2+a}(\bar{G})$.

In this paper we show that the increase of the smoothness of solutions with that of the coefficients of (1.1) and the boundary data is no longer true if $\partial \Omega$ has corners, and obtain a smoothness theorem for this case.

\section{§2. Main result}

Let $\Omega \subset R^{2}$ be a simply connected bounded domain with boundary $\partial \Omega$. For simplicity and without loss of generality we assume that $\partial \Omega$ has a single corner at the origin with interior angle $\gamma>0$. We assume that the two $\operatorname{arcs} \Gamma_{1}$ and $\Gamma_{2}$ forming the corner have the representation

$$
\begin{aligned}
& \Gamma_{1}: x_{1}=g_{2}\left(x_{2}\right) \\
& \Gamma_{2}: x_{2}=g_{1}\left(x_{1}\right)
\end{aligned}
$$

with $g_{1}$ and $g_{2}$ of class $C^{2+\alpha}$ and $g_{1}(0)=0, g_{2}(0)=0, g_{1}^{\prime}(0)=0$, and $g_{2}^{\prime}(0)=$ $\cot \gamma$.

We consider the problem [cf. (1.1)]

$$
\begin{gathered}
L u=\sum_{i, j=1}^{2} a_{i j}(x, t) u_{x_{i} x_{j}}+\sum_{j=1}^{2} a_{j}(x, t) u_{x_{j}}+b(x, t) u-u_{t}=f(x, t) \quad \text { in } G, \\
u(x, 0)=0 \quad \text { on } \bar{\Omega}, \\
\beta(x, t) u+\eta(x, t) u_{n}=0 \quad \text { on } S=(\partial \Omega \backslash\{0\}) \times(0, T],
\end{gathered}
$$

where $\beta \in C^{2+\alpha}(S), \eta \in C^{1+\alpha}(S)$, and 


$$
\beta=1, \eta=0 \text { on } \Gamma_{1}, \quad \beta=0, \eta=1 \text { on } \Gamma_{2} \text {. }
$$

If $a_{i j}, a_{j}, b, f \in C^{\alpha}(\bar{G})$, then for any bounded solution of (2.1)-(2.2) we have

$$
u \in C^{2+\alpha}\left(G_{1}\right) \cap C^{0}(\bar{G})
$$

where $G_{1}=\Omega_{1} \times(0, T]$ and $\Omega_{1}$ is any compact subregion of $\bar{\Omega}$ having positive distance from 0 .

To investigate the smoothness of solutions near the edge, we take any fixed $t_{0} \in[0, T]$ and transform the equation

$$
\sum_{i, j=1}^{2} a_{i j}\left(0, t_{0}\right) u_{x_{i x_{j}}}=0
$$

to canonical form. Then $\gamma$ at $\left(0, t_{0}\right)$ is transformed to

$$
\omega\left(t_{0}\right)=\arctan \frac{\left[a_{11}\left(0, t_{0}\right) a_{22}\left(0, t_{0}\right)-a_{12}^{2}\left(0, t_{0}\right)\right]^{1 / 2}}{a_{22}\left(0, t_{0}\right) \cot \gamma-a_{12}\left(0, t_{0}\right)} .
$$

Clearly, the value $\omega\left(t_{0}\right)$ does not depend on the particular choice of that transformation.

We now state our main result. From (2.3) it follows that it suffices to consider the smoothness of solutions near the edge.

THEOREM 1. Let $u$ be a bounded solution of (2.1), (2.2) in $\mathscr{G}_{c}=\Omega_{c}^{*} \times$ $(0, T]$, where

$$
\Omega_{c}^{*}=\{x|x \in \Omega, 0<| x \mid \leqq c\} .
$$

Assume $a_{i j}, a_{j}, b, f \in C^{\alpha}\left(\overline{\mathscr{G}}_{c}\right)$ in (2.1), where $0<\alpha<1$, and, furthermore, $\omega(t)<\frac{1}{2} \pi$ for every $t \in[0, T]$. Then $u$, as a function of $x$, satisfies

$$
u \in C^{\nu}\left(\overline{\mathscr{G}}_{c}\right) \text {, }
$$

for some $\nu \in(1,2]$.

The proof of this theorem will result from Theorem 2 (below) and will be given at the end.

\section{§3. The case of a cylindrical sector}

Let $t_{0} \in[0, T]$ be fixed and define

$$
G_{o}=\Omega_{o} \times I_{o}
$$

where 


$$
\Omega_{\sigma}=\left\{(r, \theta) \mid, 0<r \leqq \sigma, \frac{1}{2} \pi-\omega<\theta<\frac{1}{2} \pi\right\}
$$

with $\omega=\omega\left(t_{0}\right)<\frac{1}{2} \pi$ and $r, \theta$ given by $x_{1}=r \cos \theta, x_{2}=r \sin \theta$, and

$$
I_{\sigma}=\left\{t|t \in(0, T],| t-t_{0} \mid \leqq \sigma\right\} .
$$

Furthermore, let

$$
\begin{aligned}
& \Gamma_{1}=\left\{(r, \theta) \mid r \leqq \sigma, \theta=\frac{1}{2} \pi-\omega\right\}, \\
& \Gamma_{2}=\left\{(r, \theta) \mid r \leqq \sigma, \theta=\frac{1}{2} \pi\right\} .
\end{aligned}
$$

As the mixed initial-boundary conditions we take

$$
\begin{gathered}
u(x, 0)=0 \quad \text { on } \Omega_{\sigma}, \\
u\left|\Gamma_{1}=u_{n}\right| \Gamma_{2}=0, \quad 0<t \leqq T .
\end{gathered}
$$

Then we have the following result.

TheOREM 2. Let $u$ be a bounded solution of the problem (2.1), (3.1) in $G_{o}$ with $\omega<\frac{1}{2} \pi$. Suppose that $a_{i j}, a_{j}, b, f \in C^{\alpha}\left(\bar{G}_{o}\right), 0<\alpha<1$, and $a_{i j}\left(0, t_{0}\right)$ $=\delta_{i j}$. Then $u$, as a function of $x$, satisfies

$$
u \in C^{\nu}\left(\bar{G}_{a}\right),
$$

where $a<\sigma, \nu=\min (2, \pi / 2 \omega-\varepsilon)$ and $\varepsilon>0$ is arbitrarily small.

We shall obtain a proof of this theorem by first proving two lemmas in the next two sections.

\section{§4. Estimation of solutions}

LEMMA 1. Under the assumptions of Theorem 2 there exists a positive $a<\sigma$ such that in $\bar{G}_{a}$ (cf. Sec. 3)

$$
|u(x, t)| \leqq M r^{\nu},
$$

where $\nu=\min (2, \pi / 2 \omega-\varepsilon)$ and $\varepsilon>0$ is arbitrarily small.

Proof. In $G_{\sigma}$ we consider the function

$$
\begin{gathered}
v(x)=-M r^{\nu} \cos \lambda\left(\frac{1}{2} \pi-\theta\right), \\
\nu=\min \left(2, \frac{\pi}{2 \omega}-\varepsilon\right)<\frac{\pi-2 \delta}{2 \omega}=\lambda, \quad 0<\delta<\frac{1}{2} \pi .
\end{gathered}
$$

We shall prove that $v(x)$ serves as a barrier for the solution $u$ of (2.1), (3.1). We have 


$$
L v=M\left[\left(\lambda^{2}-\nu^{2}\right) \cos \lambda\left(\frac{1}{2} \pi-\theta\right)-h(x, t)\right] r^{\nu-2}+M h_{1}(x, t) r^{\nu-1}+M h_{2}(x, t) r^{\nu},
$$

where $h(x, t)$ is continuous, $h\left(0, t_{0}\right)=0$, and $h_{1}$ and $h_{2}$ are bounded. Thus for every $\varepsilon_{1}>0$ we can find an $a<\sigma$ such that in $G_{a}$,

$$
L v \geqq M\left[\left(\lambda^{2}-\nu^{2}\right) \cos \lambda\left(\frac{1}{2} \pi-\theta\right)-\varepsilon_{1}\right] r^{\nu-2} .
$$

Since for $\frac{1}{2} \pi-\omega \leqq \theta \leqq \frac{1}{2} \pi$ we have

$$
\sin \delta \leqq \cos \lambda\left(\frac{1}{2} \pi-\theta\right) \leqq 1,
$$

in $G_{a}$ we thus obtain

$$
L v \geqq M\left[\left(\lambda^{2}-\nu^{2}\right) \sin \delta-\varepsilon_{1}\right] r^{\nu-2}
$$

with arbitrarily small $\varepsilon_{1}>0$ and sufficiently small $a<\sigma$. We now take $\varepsilon_{1}<\left(\lambda^{2}-\nu^{2}\right) \sin \delta$ and use the fact that $f(x, t)$ is bounded in $G_{\sigma}$, say, $|f(x, t)| \leqq$ $K$. Consequently, by taking $M$ sufficiently large (if $\nu=2$ ) and $a$ sufficiently small (if $\nu<2$ ), we obtain in $G_{a}$

$$
L v \geqq K \geqq f(x, t) .
$$

We now want to apply in that region the maximum principle to $w=u$ $-v$. We have $L w \leqq 0$ in $G_{a}$. Furthermore, $w_{n}=0$ on $\Gamma_{2} \times I_{a}$ and $w \geqq 0$ on $\Gamma_{1} \times I_{a}$. Since $u$ is bounded in $G_{a}$, by taking $M$ sufficiently large we can make $w \geqq 0$ on

$$
\left\{(r, \theta) \mid r=a, \frac{1}{2} \pi-\omega<\theta<\frac{1}{2} \pi\right\} \times I_{a} .
$$

Hence, by the maximum principle (cf. [13]) we have $w \geqq 0$ in $\bar{G}_{a}$ with sufficiently small $a$; that is,

$$
u \geqq-M r^{\nu} \cos \lambda\left(\frac{1}{2} \pi-\theta\right) \geqq-M r^{\nu} .
$$

The other part of (4.1) can be obtained similarly. This proves Lemma 1.

\section{§5. Proof of Theorem 2}

To prove Theorem 2, we need another lemma, as follows.

Lemma 2. Let $u$ be a bounded solution of (2.1), (2.2) in $G_{\sigma}$. Suppose that $a_{i j}, a_{j}, b, f \in C^{\alpha}\left(\bar{G}_{\sigma}\right)$. Then if for some $\mu, 0<\mu \leqq 2$, and $a<\sigma$ we have

$$
|u(x, t)| \leqq M r^{\mu} \quad \text { in } \bar{G}_{a},
$$

it follows that 


$$
\left|\frac{\partial^{k} u(x, t)}{\partial x_{1}^{k_{2}} \partial x_{2}^{k-k_{1}}}\right| \leqq M_{k} r^{\mu-k} \quad \text { in } \bar{G}_{a}
$$

where $k_{1}=0,1$ or $2, k_{1} \leqq k, k=1,2$.

Proof. It suffices to indicate the basic idea since similar proofs were used in [2] and [3]. In $\Omega_{a}$ we define

$$
\begin{gathered}
D_{s}=\left\{(r, \theta) \mid 2^{-s-2} a \leqq r \leqq 2^{-s-1} a, \frac{1}{2} \pi-\omega<\theta<\frac{1}{2} \pi\right\}, \\
D_{s}^{\prime}=D_{s-1} \cup D_{s} \cup D_{s+1}, \quad s=0,1, \cdots,
\end{gathered}
$$

and set

$$
R_{s}=D_{s} \times I_{a} \quad R_{s}^{\prime}=D_{s}^{\prime} \times I_{a} .
$$

The transformation

$$
x_{i}=2^{-s} y_{i}, \quad i=1,2,
$$

transforms (2.1), (2.2) into

$$
\sum \tilde{a}_{i j} \tilde{u}_{y_{i} y_{j}}+2^{-s} \sum \tilde{a}_{j} \tilde{u}_{y_{j}}+2^{-2 s} \tilde{b} \tilde{u}-2^{-2 s} \tilde{u}_{t}=2^{-2 s} \tilde{f}, \quad \tilde{\beta} \tilde{u}+\tilde{\eta} \tilde{u}_{n}=0 .
$$

Furthermore, it maps $R_{s}$ onto $R_{0}$ and $R_{s}^{\prime}$ onto $R_{0}^{\prime}$. In $R_{0}$ and $R_{0}^{\prime}$ we apply Schauder estimates for the solution of the transformed problem to obtain

$$
\|\tilde{u}\|_{2+\alpha}^{R_{0}} \leqq C\left[\|\tilde{u}\|_{0^{\prime}}^{R^{\prime}}+2^{-2 s}\|\tilde{f}\|_{\alpha}^{R^{\prime}}\right] .
$$

This yields in $R_{s}$

$$
\left|\frac{\partial^{k} u(x, t)}{\partial x_{1}^{k_{1} \partial} \partial x_{2}^{k-k_{1}}}\right| \leqq c_{0} 2^{-(\mu-k) s},
$$

which entails the assertion of Lemma 2.

From this lemma (with $\mu=\nu$ ) we shall now obtain Theorem 2 .

Proof of Theorem 2. It is sufficient to show that

$$
\frac{\left|u_{x}(P)-u_{x}(Q)\right|}{d(P, Q)^{\nu-1}} \leqq H
$$

for any two points $P:\left(r_{1}, \theta_{1}, t_{1}\right)$ and $Q:\left(r_{2}, \theta_{2}, t_{2}\right)$ in $\bar{G}_{a}$. Here

$$
d(P, Q)^{2}=\left(x_{1}-x_{2}\right)^{2}+\left(y_{1}-y_{2}\right)^{2}+\left|t_{1}-t_{2}\right|,
$$

as usual. Without restriction, let $0 \leqq r_{2} \leqq r_{1}$. If $r_{2} \leqq r_{1} / 2$ or $\left|t_{1}-t_{2}\right|^{1 / 2} \geqq$ $r_{1} / 2$, then $d(P, Q) \geqq r_{1} / 2$ and (5.2) can be obtained using the bound (5.1) of $u_{x}$. Now let $r_{2}>r_{1} / 2$ and consider the region 


$$
S_{P}=\left\{(x, t)\left|(x, t) \in G_{a}, \frac{1}{2} r_{1} \leqq r \leqq r_{1},\right| t-t_{1} \mid \leqq \frac{1}{4} r_{1}^{2}\right\} .
$$

The transformation

$$
x_{i}=2 r_{1} z_{i} / a, \quad i=1,2,
$$

maps $S_{P}$ onto

$$
G_{P}^{\prime}=\left\{(z, t)\left|\frac{1}{4} a \leqq \rho \leqq \frac{1}{2} a,\right| t-t_{1} \mid \leqq \frac{1}{4} r_{1}^{2}\right\},
$$

where $\rho^{2}=z_{1}^{2}+z_{2}^{2}$. It transforms equation (2.1) to

$$
\sum a_{i j}^{*} u_{i i_{j}}^{*}+\frac{2 r_{1}}{a} \sum a_{j}^{*} u_{j}^{*}+\left(\frac{2 r_{1}}{a}\right)^{2} b^{*} u^{*}-\left(\frac{2 r_{1}}{a}\right)^{2} u_{t}^{*}=\left(\frac{2 r_{1}}{a}\right)^{2} f^{*}
$$

In $G_{P}^{\prime}$ and

$$
G_{P}^{\prime \prime}=\left\{(z, t)\left|\frac{1}{8} a \leqq \rho \leqq a,\right| t-t_{1} \mid \leqq \frac{1}{4} r_{1}^{2}\right\}
$$

we apply a Schauder estimate to get

$$
\left\|u^{*}\right\|_{2+\alpha}^{G_{P}^{\prime}} \leqq C\left[\left\|u^{*}\right\|_{0}^{G_{P}^{\prime \prime}}+\left(\frac{2 r_{1}}{a}\right)^{2}\left\|f^{*}\right\|_{\alpha}^{G_{P}^{\prime \prime}}\right] .
$$

As in the proof of Lemma 2 we obtain

$$
\left\|u^{*}\right\|_{2+\alpha}^{G_{P}^{\prime}} \leqq M_{2} r_{1}^{\nu}
$$

Now

$$
H_{\nu-1}^{G_{P}}\left(u_{x}\right)=\left(\frac{2 r_{1}}{a}\right)^{\nu} H_{\nu-1}^{G_{P}^{\prime}}\left(u_{z}^{*}\right)
$$

where $H_{\nu-1}^{G_{P}}\left(u_{x}\right)$ is the Hölder coefficient of the Hölder condition for $u_{x}$ in $G_{P}$ (with exponent $\nu-1$ ). From (5.3) we thus obtain

$$
H_{\nu-1}^{G_{P}}\left(u_{x}\right) \leqq M_{3}
$$

This proves Theorem 2.

\section{§6. Proof of Theorem 1. Concluding remarks}

Consider any point $\left(0, t_{0}\right), t_{0} \in[0, T]$. We straighten the boundary around $\left(0, t_{0}\right)$ by the transformation

$$
y_{1}=x_{1}-g_{2}\left(x_{2}\right), \quad y_{2}=x_{2}-g_{1}\left(x_{1}\right) .
$$

Equation (2.1) is then transformed to a parabolic equation with principal part 


$$
\sum_{i, j=1}^{2} A_{i j}(y, t) U_{y_{i} y_{j}}
$$

Clearly, (6.1) is of class $C^{2+\alpha}$ and the value of the Jacobian at $\left(0, t_{0}\right)$ is 1 . By another linear transformation we cast

$$
\sum_{i, j=1}^{2} A_{i j}\left(0, t_{0}\right) U_{y_{i} y_{j}}=0
$$

to canonical form. These two transformations map $\Omega_{c}^{*}$ in Theorem 1 onto a sector $\Omega_{\sigma}$ of angle $\omega\left(t_{0}\right)$. In $G_{\sigma}=\Omega_{\sigma} \times(0, T]$ with a suitable $\sigma>0$ the transformed functions satisfy all the conditions of Theorem 2 . Hence the conclusion of Theorem 2 applies to the transformed solution. Since the composite of those transformations is of class $C^{2+\alpha}$ and locally injective, Theorem 1 follows.

Remark 1. In [3] we were concerned with the initial Dirichlet problem for a parabolic equation with coefficients $a_{i j}$ independent of $t$. Our present results now permit us to extend our considerations in [3] for the case $n$ $=2$ as follows.

TheOREm 3. Let $u$ be a bounded solution of the initial-Dirichlet problem

$$
\begin{array}{rlrl}
L u & =f & & \text { in } G_{c} \\
u(x, 0) & =0 & & \text { on } \bar{\Omega} \\
u=0 & & \text { on } S
\end{array}
$$

with $L, \Omega$ and $S$ as in Sec. 2. Suppose that $a_{i j}, a_{j}, b, f \in C^{\alpha}\left(\bar{G}_{c}\right)$ and $\omega(t)$ $<\pi$ for every $t \in[0, T]$. Then

$$
u \in C^{\nu}\left(\bar{G}_{c}\right)
$$

for some $\nu \in(1,2]$.

To prove this, find a bound for $u$, using the idea of the proof of the above Lemma 1 and then continue as in [3].

Remark 2. If $u$ and the coefficients of the equation in Theorem 2 are extended by symmetry across $\theta=\frac{1}{2} \pi$, then in $\overline{\widetilde{G}}_{c} \backslash J$ with $\tilde{G}_{c}=\tilde{\Omega}_{c} \times I_{c}$ and

$$
\begin{aligned}
\tilde{\Omega}_{c} & =\left\{(r, \theta) \mid 0<r \leqq c, \frac{1}{2} \pi-\omega<\theta<\frac{1}{2} \pi+\omega\right\}, \\
J & =\left\{(\theta, t) \mid \theta=\frac{1}{2} \pi, t \in I_{c}\right\}
\end{aligned}
$$

the extended function satisfies an initial Dirichlet problem (instead of a mixed problem), and we may still apply the maximum principle for gener- 
alized solutions to obtain a bound for the solution. However, in Lemma 2 it is required that we have a bound for the solution everywhere. Hence the approach just mentioned would not be of help in the present case.

Remark 3. Combining Theorems 1 and 3 we obtain

Theorem 4. Let $\Omega \subset \boldsymbol{R}^{2}$ be a bounded domain whose boundary $\partial \Omega$ is a simple polygon, with sides $\Gamma_{1} \cdots, \Gamma_{p}$ of class $C^{2+\alpha}$ and vertices $P_{j}=\Gamma_{j} \cap$ $\Gamma_{j+1}, j=1, \cdots, p,\left(\Gamma_{p+1}=\Gamma_{1}\right)$ with angles $\gamma_{j}$ such that for the corresponding $\omega_{j}=\omega\left(P_{j}, t\right)$ we have

$$
\begin{aligned}
\omega_{j} & =\frac{1}{2} \xi_{j}(t)\left(\kappa_{j}+\kappa_{j+1}\right), \quad 0<\xi_{j}<\pi, \\
\kappa_{j} & =0 \text { or } 1, \quad \kappa_{j}+\kappa_{j+1} \neq 0, \quad j=1, \cdots, p\left(\kappa_{p+1}=\kappa_{1}\right) .
\end{aligned}
$$

Let $u$ be a bounded solution of the problem

$$
\begin{aligned}
& L u=f \text { in } \Omega \times I, \quad I=(0, T], \\
& u(x, 0)=0 \quad \text { in } \bar{\Omega}, \\
& \kappa_{j} u+\left(1-\kappa_{j}\right) u_{n}=0 \quad \text { on } \Gamma_{j} \times I,
\end{aligned}
$$

with $L$ as in (2.1) and $a_{i j}, a_{j}, b, f \in C^{\alpha}(\bar{\Omega} \times \bar{I}), 0<\alpha<1$. Then

$$
u \in C^{2+\alpha}\left(\bar{\Omega}_{1} \times \bar{I}\right)
$$

where $\bar{\Omega}_{1}$ is any compact subregion of $\bar{\Omega}$ with positive distance from the corners, and

$$
\begin{aligned}
& u \in C^{\nu_{j}}\left(\left[\bar{\Omega} \cap N_{j}\right] \times \bar{I}\right) \\
& \nu_{j}=\min _{t \in I}\left(2, \pi / \xi_{j}(t)-\varepsilon\right),
\end{aligned}
$$

where $\varepsilon>0$ is arbitrarily small and $N_{j}$ is a sufficiently small closed disk centered at $P_{j}$.

The proof is obvious; indeed, for $\kappa_{j}+\kappa_{j+1}=1$ (mixed data) it follows from Theorem 1 and for $\kappa_{j}+\kappa_{j+1}=2$ (Dirichlet data) from Theorem 3 .

Acknowledgement. We want to thank the Reviewer for valuable comments which entailed a substantial improvement of this paper.

\section{REFERENCES}

[1] S. Agmon, A. Douglis and L. Nirenberg, Estimates near the boundary for solutions of elliptic partial differential equations satisfying general boundary conditions, I., Comm. Pure Appl. Math., 12 (1959), 623-727. 
[2] A. Azzam, On Dirichlet's problem for elliptic equations in sectionally smooth $n$ dimensional domains, SIAM J. Math. Anal., 11 (1980), 248-253.

[ 3 ] A. Azzam and E. Kreyszig, On parabolic equations in $n$ space variables and their solutions in regions with edges, in press.

[4] H. S. Carslaw and J. C. Jaeger, Conduction of heat in solids, Clarendon Press, Oxford (1959).

[5] A. Friedman, Boundary estimates for second order parabolic equations and their applications, J. Math. Mech., 7 (1958), 771-791.

[6] D. Gilbarg and N. S. Trudinger, Elliptic partial differential equations of second order, Springer, Berlin etc., 1977.

[7] P. Grisvard, Behavior of the solutions of an elliptic boundary value problem in a polygonal or polyhedral domain; in: Numerical solutions of partial differential equations III (ed. B. Hubbard), Academic Press, New York (1976).

[8] Z. Itô, A boundary value problem of partial differential equations of parabolic type, Duke Math. J., 24 (1957), 299-312.

[ 9 ] L. I. Kamynin and V. N. Maslennikova, Boundary estimates for the solution of the third boundary-value problem for a parabolic equation, Dokl. Akad. Nauk SSSR, 153 (1963), 526-529.

[10] V. A. Kondrat'ev, Singularities of a solution of Dirichlet's problem for a second order elliptic equation in the neighborhood of an edge, Diff. Uravnen., 13 (1977), 2026-2033.

[11] $P$. Laasonen, On the degree of convergence of discrete approximations for the solutions of the Dirichlet problem, Ann. Acad. Fenn. Ser. A. I. No. 246 (1957), $19 \mathrm{pp}$.

[12] O. A. Ladyženskaja, V. A. Solonnikov and N. Ural'ceva, Linear and quasi-linear equations of parabolic type, American Mathematical Society, Providence, R. I. 1968.

[13] M. H. Protter and H. F. Weinberger, Maximum principles in differential equations, Prentice-Hall, Englewood Cliffs, N. J. (1967).

[14] E. A. Volkov, Differentiability properties of solutions of boundary value problems for the Laplace equation on a polygon, Proc. Steklov Inst. Math., 77 (1965), 127159.

[15] N. V. Zitaraŝu, Schauder estimates and solvability of general boundary problems for general parabolic systems with discontinuous coefficients, Soviet Math. Dokl., 7 (1966), 952-956.

Department of Mathematics

University of Windsor

Windsor, Ontario, Canada 